\title{
Hybrid mode-lock InGaAsP/InP MQW laser as a multiwavelength source for WDM
}

T. R. Chen, Roger M. P. Koumans, D. Eliyahn, Randal A. Salvatore, Amnon Yariv

T. R. Chen, Roger M. P. Koumans, D. Eliyahn, Randal A. Salvatore, Amnon Yariv, "Hybrid mode-lock InGaAsP/InP MQW laser as a multiwavelength source for WDM," Proc. SPIE 3547, Semiconductor Lasers III, (19 August 1998); doi: 10.1117/12.319640

SPIE. Event: Photonics China '98, 1998, Beijing, China 


\title{
Hybrid Mode-Lock InGaAsP/InP MQW Laser As a Multi-wavelength Source for WDM
}

\author{
T.R. Chen, R.G.M.P. Koumans, D. Eliyahn, R.A. Salvatore, and A. Yariv \\ Department of Applied Physics 128-95 \\ California Institute of Technology \\ 1201 E. California Blvd., Pasadena, CA 91125 \\ Tel: (626)395-3982 Fax: (626)405-0928
}

\begin{abstract}
A hybrid mode-locked semiconductor laser was demonstrated as a multi-wavelength light source for WDM networks. The laser simultaneously provides over $50 \mathrm{WDM}$ channels spaced at $21.8 \mathrm{GHz}$ $(0.17 \mathrm{~nm})$. Channel spacing is temperature independent and is determined by the modulating frequency applied to the absorber section of the laser, thus is very stable. The wavelength of the channels can be slightly tuned by injection current and/or temperature. We demonstrate dropping a WDM channel at a network node by using a Fiber Bragg Grating combined with a fiber coupler.
\end{abstract}

Key words: Wavelength division multiplexing, mode locked laser, Fiber Bragg Grating

\section{INTRODUCTION}

Wavelength Division Multiplexing (WDM) is now widely acknowledged as one of the most promising technology to meet the challenge of the ever increasing demand on telecommunication capacity, and is being rapidly deployed. WDM systems utilize the large bandwidth available from an optical fiber very effectively by launching multiple closely spaced optical channels into a single fiber, each modulated at high bit-rate, e.g., $2.5 \mathrm{~Gb} / \mathrm{s}$ or $10 \mathrm{~Gb} / \mathrm{s}$. The use of multiple wavelengths also offers another degree of freedom to overcome the bandwidth limitation placed either by high speed electronics available or by fiber dispersion, as in the case of using a single wavelength source.

One of the key components of a WDM system is a multi-wavelength light source. Wavelength stability, precise control of channel spacing, low crosstalk, and cost effectiveness are some of the important requirements for WDM light sources.

A large number of multi-wavelength sources have been proposed and demonstrated. Some examples are as follows: a set of discrete DFB lasers, each has a desired wavelength ${ }^{1}$, monolithically integrated semiconductor laser array with each element having slightly different wavelength ${ }^{2}$, spectral slicing of devices with a broad optical emission spectrum, for example, light emitting diode ${ }^{3}$, or semiconductor optical amplifier ${ }^{4}$, a multi-frequency laser with a waveguide grating router as an intracavity element to lock all wavelengths at a specifically determined channel spacing 5 , amplification of spontaneous emission from a Fabry-Perot (F-P) laser biased below threshold6, super continuum (SC) generation in optical fiber7, tunable DFB lasers, and mode-locked semiconductor laser 8,9 . Among all the WDM sources proposed to date, the mode-locked semiconductor laser has the following unique advantages: Tens to hundreds different wavelengths can be generated simultaneously with equally spaced channels. The channel spacing is basically determined by the laser cavity length. The application of an RF signal to the saturable absorber section of the mode-locked laser makes the channel spacing temperature independent and very stable. Fine tuning of the channel spacing can be accomplished by 
slightly changing the RF modulation frequency from the cavity repetition rate. This kind of WDM source is well suitable for the network infrastructure where the multi-wavelength source is located at the central node and different wavelength is individually modulated at each network node 10 .

\section{LASER STRUCTURE, FABRICATION, AND CHARACTERISTICS}

In this work, the hybrid mode-locking of a semiconductor laser was demonstrated. The laser was a monolithic integration of a long gain section and a short saturable absorber. A schematic drawing of the mode-locked laser is shown in Fig. 1. The active layer consists of four compressively strained quantum wells, grown by MOCVD. The device uses a buried heterostructure to form reverse biased junctions as current blocking layers and also to provide lateral optical confinement. The device length is $2010 \mu \mathrm{m}$ while the absorber section is only $\sim 100 \mu \mathrm{m}$. The isolation region between the gain and the absorber section is approximately $15 \mu \mathrm{m}$. Electrical isolation between the two sections is $1.5 \mathrm{k} \Omega$. No coatings were applied to the end facets of the laser. The round trip repetition frequency, calculated from the cavity length is $\sim 21.8 \mathrm{GHz}$. The laser showed excellent static characteristics. Threshold current of the laser with unbiased absorber is as low as $\sim 35 \mathrm{~mA}$, despite the very long cavity length. The slope efficiency is $\sim 0.055 \mathrm{~mW} / \mathrm{mA}$. The emission wavelength is $\sim 1550 \mathrm{~nm}$.

In order to make the absorber section suitable for high frequency modulation, the parasitic capacitance has to be reduced. Two factors contribute to the parasitic capacitance, one is the distributed capacitance from the reverse biased block junction, the other is the contact bonding pad capacitance (contact RC).

In our laser, an isolated narrow mesa $(\sim 10 \mu \mathrm{m}$ in width) was formed by etching a pair of channels along the active region. This reduced the capacitance caused by the reverse-biased blocking layers. A thick $\mathrm{SiO}_{2}$ layer was deposited on the top surface of the device to isolate the contact. An air bridge contact configuration has been adopted to provide electrical contact to the absorber section 11 . This further reduced the parasitic capacitance caused by the metal/ $\mathrm{SiO}_{2} /$ semiconductor interface compared with a continuous contact configuration in which the contact metal continuously covers the bottom surface of the isolation channel.

The monolithic device was mounted on a custom high frequency mount consisting of a kconnector and a $50 \Omega$ co-planner. This mount allows RF modulation up to $40 \mathrm{GHz}$. The laser was not impedance matched to the co-planner. The frequency response of the absorber (see Fig. 2) was measured using a network analyzer and a high speed photodetector. The gain section was pumped at $130 \mathrm{~mA}$ and the absorber was DC biased at $-0.26 \mathrm{~V}$. A cavity resonance peak occurs at around $21.8 \mathrm{GHz}$.

\section{MODE-LOCKING OF THE LASER}

The laser was first passively mode-locked. In this case, the gain and absorber section were DC biased under the conditions mentioned above. No RF signal was applied to the absorber. The RF spectrum of the light output was measured with a high speed detector and an electrical spectrum analyzer (see Fig. 3, solid line). The passive mode-locking resonance occurred at $21.805 \mathrm{GHz}$ while the $3 \mathrm{~dB}$ linewidth of the electrical spectrum was $9 \mathrm{MHz}$.

In order to stabilize the channel spacing and make it temperature independent, we hybridly modelocked the laser by applying an RF signal to the reverse biased absorber. The network analyzer was used as an RF source and the modulating power was boosted to $+17 \mathrm{dBm}$ with an RF amplifier. The RF 
spectrum of the hybridly mode-locked laser is shown in Fig. 3 for two different RF modulation frequencies: $21.764 \mathrm{GHz}$ and $21.83 \mathrm{GHz}$ (dash lines). Contrast ratio of peak to background noise for hybrid mode-locking is larger than $20 \mathrm{~dB}$. The linewidth of the RF spectrum was reduced to $400 \mathrm{KHz}$. A locking range of $66 \mathrm{MHz}$ was observed around the passive mode-locking resonance. Over $50 \mathrm{WDM}$ channels spaced at $21.8 \mathrm{GHz}$ were demonstrated. Hybrid mode-locking of the device was also realized with an RF signal half the frequency of the cavity round trip rate $(10.9 \mathrm{GHz})$. This sub-harmonic modelocking eased the need for a very high frequency RF source. Under this condition, the laser remained mode-locked at $21.8 \mathrm{GHz}$ without any noticeable change of the RF spectrum and locking range.

\section{EXPERIMENT OF DROPPING A WDM CHANNEL}

Using a Fiber Bragg Grating, we demonstrated the principle of dropping a WDM channel. The experiment set up is sketched in Fig. 4. The incoming multi-wavelength signal from the mode-locked laser is split at the $85 / 15$ optical coupler. $85 \%$ optical power is connected to a commercially available QPS/Bragg Fiber Grating. The WDM channel of interest that needs to be dropped is reflected off the Bragg Grating back into the coupler and is available at the backward $15 \%$ output port of the coupler. All the other channels are available at the output of the fiber grating. The complete WDM spectrum (before dropping) can be measured at the forward $15 \%$ output port of the coupler.

The center wavelength of the Fiber Bragg Grating is $1539.3 \mathrm{~nm}$, the peak reflectivity is $93 \%$, and the FWHM is $0.21 \mathrm{~nm}(26.6 \mathrm{GHz})$. In order to have the wavelength of one of the WDM channels coincide with the center wavelength of the Fiber Bragg Grating, we can fine-tune the whole wavelength continuum of the mode-locked laser by changing either the temperature or the drive current of the gain section. In our case, the laser was temperature stabilized and wavelength was tuned by changing the injection current into the gain section. It was observed when the injection current changed from $100 \mathrm{~mA}$ to $160 \mathrm{~mA}$, the spectrum of the mode-locked laser could be tuned over one channel spacing $(0.17 \mathrm{~nm})$. Within this range, the laser stayed hybridly mode-locked with a background noise suppression ratio of more than $20 \mathrm{~dB}$.

The optical spectrum of all three outputs of the setup are shown in Fig. 5. The spectrum of the dropped channel and the change of the WDM signal spectrum after one channel was dropped can be seen clearly.

\section{SUMMARY}

We demonstrate the use of an air-bridge type hybridly mode-locked laser as a WDM source. Channel spacing is temperature independent and change of injection current provides fast and accurate wavelength tuning. Sub-harmonic mode-locking has also been accomplished. A method of dropping a channel using a Fiber Bragg Grating and fiber coupler has been demonstrated.

\section{REFERENCES}

1. Toba, K. Oda, K. Nakanishi, N. Shibata, K. Nosu, N. Takato, and M. Fukuda, "A 100-channel optical FDM transmission/distribution at $622 \mathrm{Mb} / \mathrm{s}$ over $50 \mathrm{~km}$," J. Lightwave Technol., vol. 8, no. 9, pp. 1396-1401, Sept. 1990.

2. C.E. Zah, B. Pathak, M. R. Amersfoot, F. Favire, P. S. D. Lin, N. C. Andreadakis, A. Rajhel, R. Bhat, C. Caneau, and M.A. Koza, and L. Curtis, "High power 10-wavelength DFB laser arrays with 
integrated combiner and optical amplifier," 15th IEEE International Semiconductor Laser Conference, 13-18 October 1996, Conference Digest, paper W1.4, pp. 131.

3. K. Y. Liou, J. B. Stark, U. Koren, E. C. Burrows, J. L. Zyskind, and K. Dreyer, "System performance of an eight-channel WDM local access network employing a spectrum sliced and delay-line-multiplexed LED source," IEEE Photon. Technol. Lett., vol. 9, no. 5, pp. 696-698, May 1997.

4. D. D. Sampson and W. T. Holloway, " $100 \mathrm{~mW}$ spectrally-uniform broad-band ASE source for spectrum-sliced WDM systems,” Electron. Lett., vol. 30, no. 19, pp. 1611-1612, Sept. 1994.

5. R. Monnard, C. R. Doerr, C. H. Joyner, M. Zirngibl, and L. W. Stulz, "Direct modulation of a multifrequency laser up t 16 x $622 \mathrm{Mb} / \mathrm{s}$," IEEE Photon. Technol. Lett., vol. 9, no. 6, pp. 815-817, June 1997.

6. W. T. Holloway, A. J. Keating, and D. D. Sampson, "Multiwavelength source for spectrum-sliced WDM access networks and LAN's," IEEE Photon. Technol. Lett., vol, 9, no. 7, pp. 1014-1016, July 1997.

7. T. Morioka, K. Mori, S. Kawanishi, and M. Saruwatari, "Multi-WDM-channel, Gbit/s pulse generation from a single laser source utilizing LD-pumped supercontinuum in optical fibers, "IEEE Photon. Technol. Lett., vol. 6, no. 3, pp. 365-368, Mar. 1994.

8. D. Eliyahu, A. Yariv, and R. A. Salvatore, 'Broader, flatter optical spectra of passively mode-locked semiconductor lasers for a wavelength-division multiplexing source," Appl. Opt., vol. 36, no. 15, pp. 3430-3434, May 1997.

9. H. Sanjoh, H. Yasaka, Y. Sakai, K. Sato, H. Ishii, and Y. Yoshikuni, "Multiwavelength light source with precise frequency spacing using a mode-locked semiconductor laser and an arrayed waveguide grating filter," IEEE Photon. Technol. Lett., vol. 9, no. 6, pp. 818-820, June 1997.

10. M. Sharma, H. Ibe, and T. Ozeki, "WDM ring network using a centralized multiwavelength light source and add-drop multiplexing filters," J. Lightwave Technol., vol. 15, no. 6, pp. 917-929, June 1997.

11. T. R. Chen, P. C. Chen, C. Gee, and N. Bar-Chaim, "A high-speed InGaAsP/InP DFB laser with an air-bridge contact configuration," IEEE Photon. Technol. Lett., vol. 5, no. 1, pp. 1-3, Jan. 1993. 


\section{Figure Captions}

Fig. 1 Schematic view of the mode-locked laser showing gain and absorption section, isolated mesa and air-bridge.

Fig. 2 Small signal modulation response of the air-bridge type mode-locked laser showing cavity resonance peak at around $21.8 \mathrm{GHz}$.

Fig. 3 Electrical spectrum of the mode-locked pulse train for (a) passive mode-locking, (b) hybrid mode-locking at $21.764 \mathrm{GHz}$, (c) hybrid mode-locking at $21.83 \mathrm{GHz}$.

Fig. 4 Schematic setup of a WDM dropping system showing a 85/15 coupler and a fiber bragg grating drop filter.

Fig. 5 Optical spectra of (a) all WDM channels, (b) dropped channel, (c) remaining channels. Note that all spectras have arbitrary referral levels. 




Fig. 1 


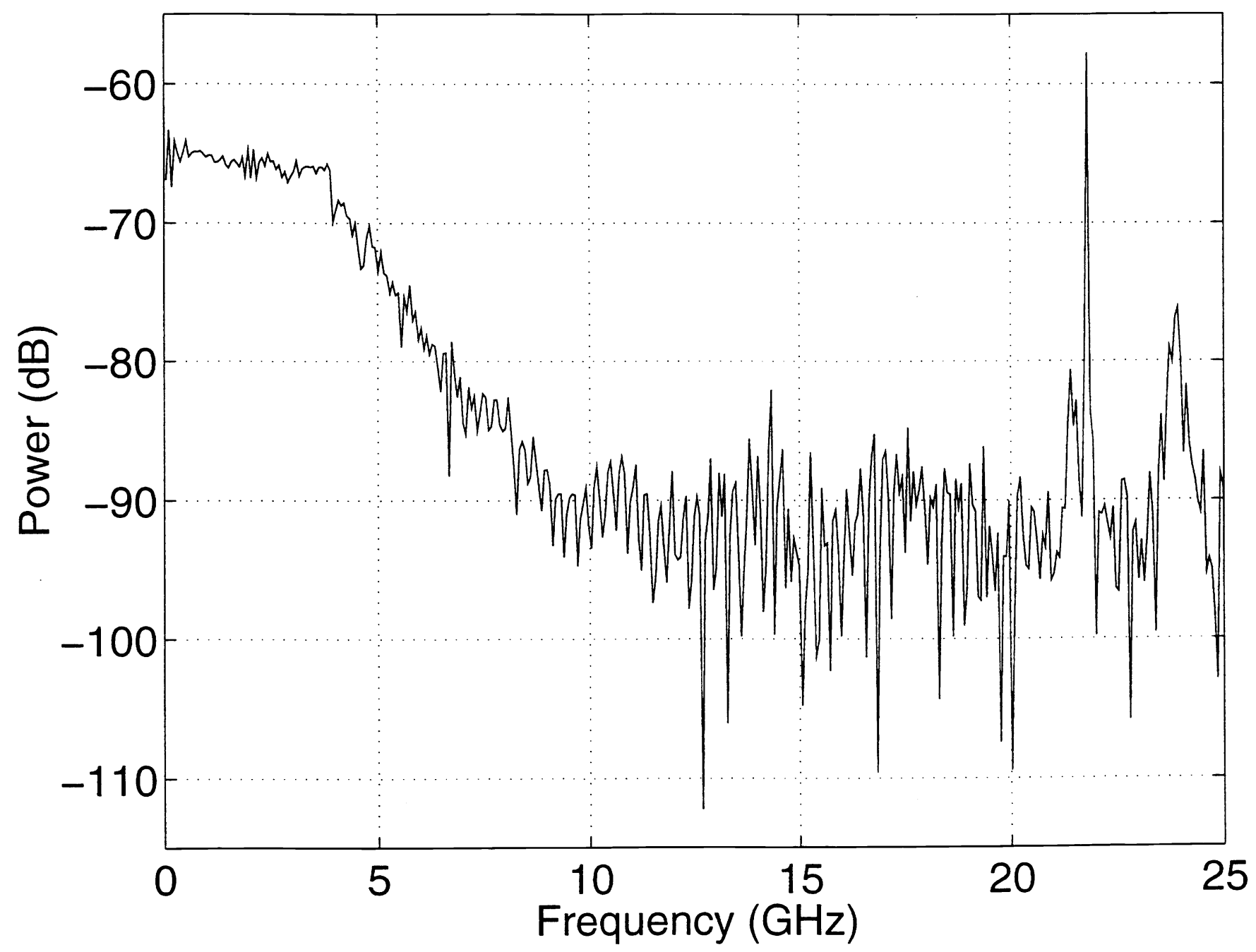

Fig. 2 


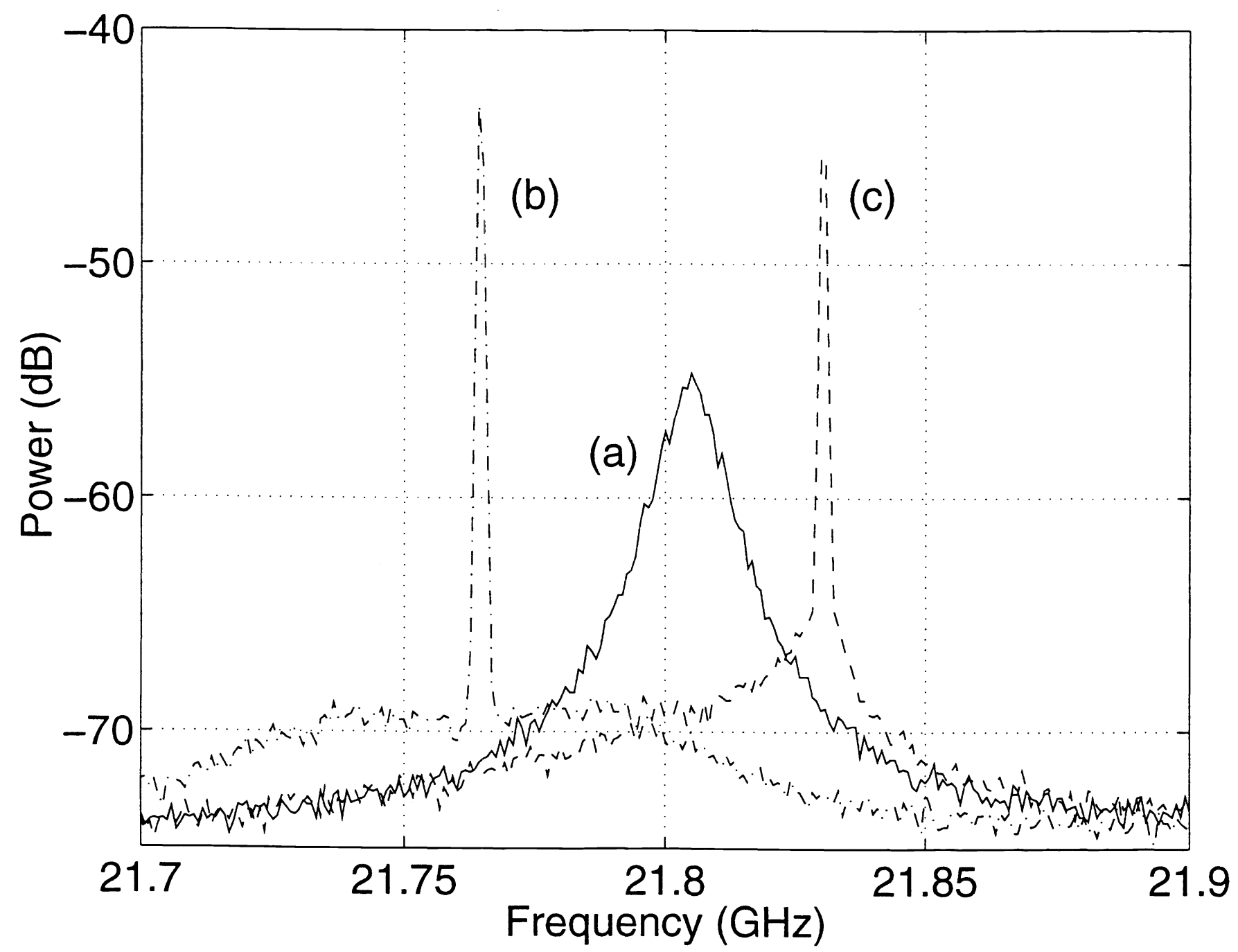

Fig. 3 


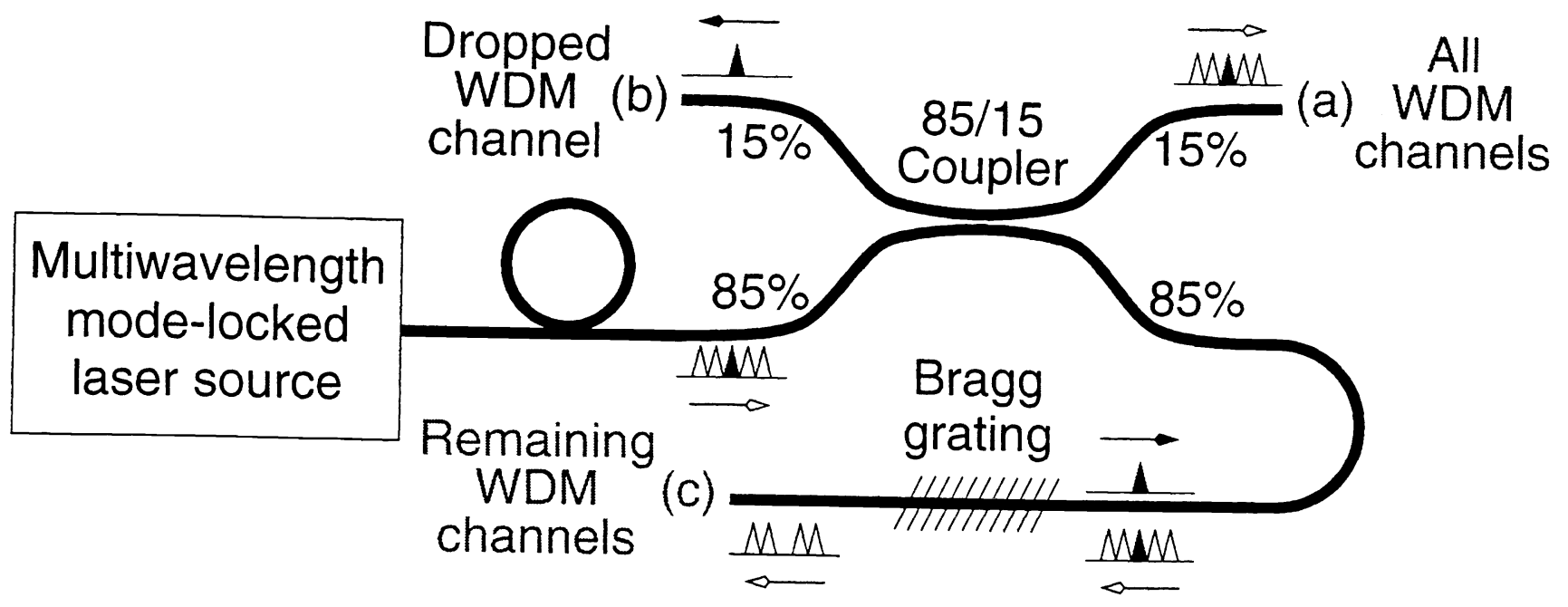

Fig. 4 


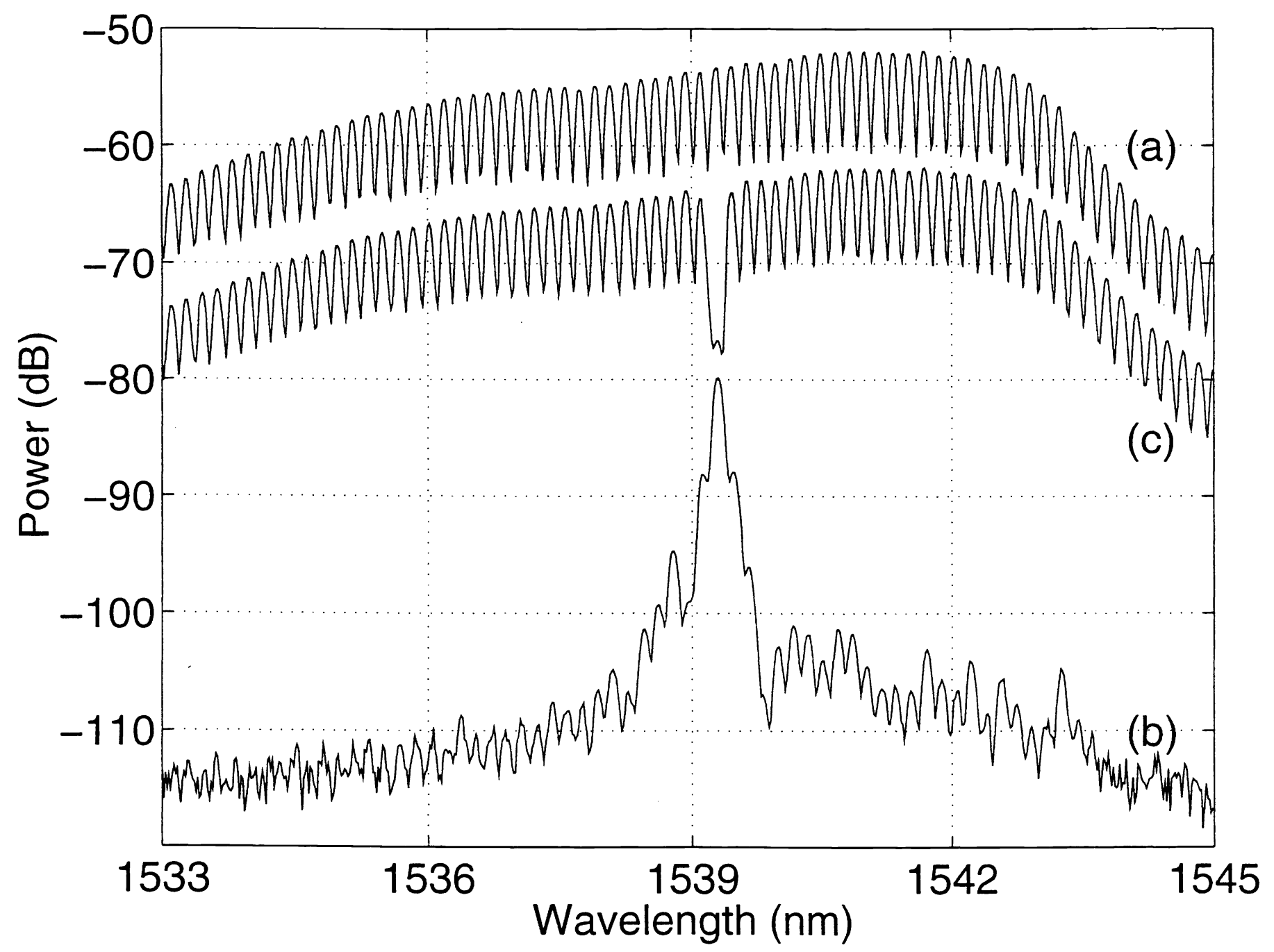

Fig. 5 